\title{
Real-World Utilisation and Bleed Rates in Patients with Haemophilia B Who Switched to Recombinant Factor IX Fusion Protein (rIX-FP): A Retrospective International Analysis
}

\author{
Cedric Hermans · Renato Marino - Catherine Lambert • \\ Sarah Mangles · Patrick Sommerer • Vincent Rives • Geraldine Maro • \\ Giuseppe Malcangi
}

Received: January 27, 2020 / Published online: April 24, 2020

(c) The Author(s) 2020

\section{ABSTRACT}

Introduction: Despite the well-documented benefits of prophylaxis, treatment burden is still a barrier to adherence in patients with haemophilia. An extended half-life fusion protein linking recombinant FIX (rFIX) with human albumin (rIX-FP) has been developed for the treatment of patients with haemophilia B and is indicated for dosing up to every 14 days. This analysis evaluated real-world outcomes in

Digital Features To view digital features for this article go to https://doi.org/10.6084/m9.figshare.11974071.

C. Hermans $(\varangle) \cdot$ C. Lambert

Haemostasis and Thrombosis Unit, Division of

Haematology, Cliniques universitaires Saint-Luc,

Université catholique de Louvain, Brussels, Belgium

e-mail: cedric.hermans@uclouvain.be

R. Marino · G. Malcangi

Hemophilia and Thrombosis Center, University

Hospital of Bari, Bari, Italy

S. Mangles

North Hampshire Haemophilia Centre, Basingstoke,

UK

P. Sommerer

CSL Behring, Hattersheim, Germany

V. Rives

CSL Behring, Paris, France

G. Maro

Adivo Associates, San Francisco, CA, USA patients switching to rIX-FP from the previous FIX product in Italy, Belgium and the UK.

Methods: Anonymised chart data were collected from the pre-existing medical records of patients with haemophilia B between May and September 2018. Patients were included in the analysis if they had been treated with rIX-FP for $\geq 8$ weeks. Data were compared between rIX-FP and the patient's prior FIX product.

Results: Twenty-three HTCs from Italy $(n=13)$, Belgium $(n=3)$ and the UK $(n=7)$ provided data for 84 male patients, $92.8 \%$ of which had severe haemophilia B. The majority of patients were previously on prophylactic regimens with their prior FIX product (Italy, 44/49; Belgium, 7/10; UK, 22/25). The switch to prophylaxis with rIX-FP led to reductions in mean annualised bleeding rate of $94.3 \%$ in Italy, $93.9 \%$ in Belgium and $67.7 \%$ in the UK compared with prior FIX prophylaxis. Overall, $41 \%$ of patients experienced zero spontaneous bleeds prior to switching, compared with $88 \%$ following the switch to rIX-FP. The majority of patients had a reduction in dosing frequency following the switch, with $98.6 \%$ of patients dosing once weekly or less frequently compared with $9.6 \%$ of patients dosing at this frequency with their prior FIX. Mean weekly FIX consumption was reduced compared with prior FIX prophylaxis.

Conclusion: This retrospective review of realworld evidence demonstrated that switching to rIX-FP from prior FIX was associated with 
improved haemostatic efficacy and reduced factor consumption in patients with haemophilia B from Italy, Belgium and the UK.

\section{PLAIN LANGUAGE SUMMARY}

While clinical trials provide robust evidence as to the effectiveness and safety of a new drug, they are tightly controlled and so may not reflect some of the issues that may be discovered in clinical practice. Therefore, real-world analyses are important to determine how a product performs in patients in everyday settings. This study looked at the use of an extended half-life fusion protein linking recombinant FIX (rFIX) with human albumin (rIX-FP), which was designed to allow longer dosing intervals, in patients with haemophilia B in Italy, Belgium and the UK, and compared this with the patients' previous FIX product. Anonymous patient chart data were collected from participating centres and analysed in terms of bleeding rate, factor usage and dosing frequency for rIX-FP and previous FIX product. The results showed that after switching to rIX-FP, patients experienced lower bleeding rates, lower factor usage and less frequent dosing regimens compared with their previous FIX product. This is the first analysis to assess the real-world clinical benefits of switching to prophylaxis with rIX-FP from a prior FIX product in Italy, Belgium and the UK. This study further strengthens the results seen in clinical trials with rIX-FP, confirming that the effective bleeding prevention demonstrated in clinical trials is consistent with that seen in patients in real-world clinical practice.

Keywords: Albutrepenonacog alfa; Belgium; Factor IX; Haemophilia B; Italy; Medical records; Recombinant fusion proteins; United Kingdom

\section{Key Summary Points}

Why carry out this study?

Clinical trials are important to generate the robust efficacy and safety data required for subsequent approval of new therapies; however, when evaluating FIX products in clinical practice, real-world utilisation and outcomes also need to be considered.

The aim of this analysis was to evaluate real-world outcomes in patients switching to rIX-FP from the previous FIX product in Italy, Belgium and the UK.

\section{What was learned from this study?}

This study demonstrated that patients experienced reduced bleeding rates, factor consumption and dosing intervals following the switch to rIX-FP compared with their previous FIX product.

This real-world analysis further strengthens the rIX-FP clinical trial data, showing that the excellent haemostatic efficacy demonstrated in clinical trials is consistent with that seen in real-world clinical practice.

\section{INTRODUCTION}

Haemophilia B is an X-linked, recessive bleeding disorder resulting in an absence or deficiency of coagulation factor IX (FIX) [1]. A deficiency in FIX can lead to abnormal or uncontrollable bleeding events, of which the frequency and severity are dependent on residual clotting factor levels [1]. The management of haemophilia B occurs primarily through the administration of FIX replacement concentrates [1]. In addition to reducing the risk of bleeding, prophylaxis with FIX concentrates has been shown to preserve joint function and is considered to be the optimal therapy for patients with severe haemophilia [2]. Optimal prophylaxis protocols 
take into account the patient's pharmacokinetic (PK) profile, bleeding pattern and musculoskeletal condition [3].

Standard half-life (SHL) FIX products require frequent intravenous infusions (2-3 times weekly) due to the short half-life (18-36 h) $[1,4,5]$. Despite the well-documented longterm benefits of prophylaxis, treatment burden is still a primary barrier to adherence in patients with haemophilia [6]. This can have a detrimental impact on patients' quality of life, which may lead to delayed adoption of prophylactic therapy and suboptimal treatment adherence [4, 7-9]. A survey of haemophilia patients and parents of children with haemophilia in Germany, Austria and Switzerland has shown that reducing treatment burden by decreasing the frequency of factor infusions was one of their most important unmet needs [10].

The most commonly prescribed SHL FIX product in Italy, Belgium and the UK is nonacog alfa [Benefix ${ }^{\circledR}$ (Pfizer Inc.)]. Extended half-life (EHL) recombinant FIX (rFIX) products with improved PK properties have been developed and provide some patients with the potential to decrease their dosing frequency whilst maintaining or reducing bleeding frequency. EHL products allowing extended dosing intervals may improve treatment adherence in some patients, potentially overcoming some of the disadvantages associated with frequent prophylaxis regimens using standard-acting products $[9,11,12]$. These EHL rFIX products include rIX-FP (albutrepenonacog alfa; IDEL$\mathrm{VION}^{\circledR}$, CSL Behring), a recombinant fusion protein linking human FIX with human albumin [13, 14]; rFIXFc (eftrenonacog alfa; Alprolix ${ }^{\circledR}$, Biogen), a recombinant FIX Fc fusion protein $[15,16]$; and N9-GP (nonacog beta pegol; Refixia ${ }^{\circledR}$, Novo Nordisk), a recombinant, glycoPEGylated FIX protein $[17,18]$.

The PROLONG-9FP clinical trial programme has demonstrated that rIX-FP provides excellent haemostatic efficacy in patients on an extended dosing interval, with a median annualised spontaneous bleeding rate (AsBR) and annualised joint bleeding rate (AjBR) of 0 [14]. Clinical trials are important to generate the robust efficacy and safety data required for subsequent approval of new therapies; however, when evaluating FIX products in clinical practice, real-world utilisation and outcomes also need to be considered. Patient response to treatment can be measured in a number of ways, but the most frequently used are annualised bleeding rate (ABR) and AsBR. Additionally, outcomes such as infusion frequency may give an indication of patient satisfaction with their treatment regimen and consumption outcomes may have important implications for the socio-economic burden of haemophilia.

The aim of this analysis was to determine real-world ABR, FIX dosing frequency and consumption in patients switching to prophylaxis with rIX-FP from their previous SHL FIX product in three European countries (Italy, Belgium and the UK).

\section{METHODS}

\section{Study Conduct}

This analysis involved a retrospective review of anonymised data from the pre-existing medical records of patients with haemophilia B collected between May and September 2018. Centres and treating physicians were unaware of the study sponsor and the study obtained only pre-existing data from patients who were never identified to researchers; therefore, informed consent was not required. The study was submitted to an Institutional Review Board and was determined to be exempt under category 4 'Secondary research for which consent is not required'.

Data obtained from patients' clinical charts included: age, sex, severity of haemophilia B; treatment regimen (prophylaxis for rIX-FP and prophylaxis or on demand for prior FIX products); dosing and infusion frequency on rIX-FP and prior FIX products; weight at time of most recent prescription of rIX-FP and prior FIX product; and the number of bleeds during the observation period for rIX-FP and prior FIX products.

Prophylaxis was defined as treatment to prevent bleeding through regular infusions over the time period analysed in this study. All patients receiving infusions to treat a bleed or 
for peri-operative management were defined as receiving on-demand therapy.

\section{Study Patients}

To be eligible for inclusion in the analysis, adult and paediatric haemophilia $B$ patients receiving treatment in haemophilia treatment centres (HTCs) in Italy, Belgium and the UK must have received at least 8 weeks of treatment with rIX-FP. In line with product labelling, adult and adolescent patients (collectively referred to as the adult group) were defined as those $\geq 12$ years, while paediatric patients were $\leq 11$ years. Patient selection was made at the discretion of the physician. The study obtained only pre-existing data from patients who were never identified to researchers and therefore did not require informed consent.

\section{Study Outcomes}

The target sample size was approximately 85 patients in all three countries combined, which represented roughly $40 \%$ of the estimated patient population treated with rIX-FP during the period of data collection in the considered countries. Primary outcome measures were bleeding rates and consumption of replacement factor in patients receiving prophylaxis with rIX-FP compared with the prior product. ABRs were calculated by annualising the number of bleeds experienced by a patient over a period of at least 8 weeks on rIX-FP and the prior FIX product. Patients with an unknown number of bleeds were not included in this analysis. Prescribed dosing and injection frequency for patients treated with rIX-FP and prior FIX product were used to calculate FIX consumption (normalised to $\mathrm{IU} / \mathrm{kg} /$ week to allow for interand intra-patient comparison by type and brand). Cost per success was estimated using pricing information available in public sources (PriceCentric) and considering nonacog alfa as the pre-switch product. Success was defined as patients experiencing zero spontaneous bleeding episodes.

\section{RESULTS}

Twenty-three HTCs from Italy $(n=13)$, Belgium $(n=3)$ and the UK $(n=7)$ provided data for a total of 84 patients treated with rIX-FP for at least 8 weeks.

\section{Patient Characteristics}

All patients were male (mean ages 28.0, 33.4 and 33.8 years in Italy, Belgium and the UK, respectively) and $92.8 \%$ of patients had severe haemophilia B (Table 1). The majority of patients in all three countries were on prophylactic regimens with their prior FIX product

Table 1 Patient demographics

\begin{tabular}{llll}
\hline & $\begin{array}{l}\text { Italy } \\
(\boldsymbol{n}=49)\end{array}$ & $\begin{array}{l}\text { Belgium } \\
(\boldsymbol{n}=\mathbf{1 0})\end{array}$ & $\begin{array}{l}\text { UK } \\
(\boldsymbol{n}=\mathbf{2 5})\end{array}$ \\
\hline Age, years & $28.0 \pm 15.1$ & $33.4 \pm 17.3$ & $33.8 \pm 16.4$ \\
Weight, kg & $60.4 \pm 20.3$ & $66.4 \pm 5.3$ & $70.5 \pm 15.8$ \\
$\begin{array}{l}\text { Severity } \\
\text { Severe, } n \text { (\%) }\end{array}$ & $48(98.0)$ & $7(70.0)$ & $23(92.0)$ \\
$\begin{array}{l}\text { Moderate/ } \\
\text { mild, } n(\%)\end{array}$ & $1(2.0)$ & $3(30.0)$ & $2(8.0)$ \\
$\begin{array}{l}\text { Current regimen } \\
\text { Prophylaxis, } \\
n(\%)\end{array}$ & $49(100)$ & $10(100)$ & $25(100)$ \\
$\begin{array}{l}\text { On demand, } \\
n(\%)\end{array}$ & $0(0)$ & $0(0)$ & $0(0)$
\end{tabular}

Prior regimen

Prophylaxis, $\quad 44(89.8) \quad 7(70.0) \quad 22(88.0)$

$n(\%)$

On demand, $5(10.2) \quad 3(30.0) \quad 3(12.0)$

$n(\%)$

Prior drug used, $n$ (\%)

\begin{tabular}{llll} 
pdFIX & $8(16.3)$ & $0(0)$ & $1(4.0)$ \\
Nonacog alfa & $41(83.7)$ & $10(100)$ & $24(96.0)$ \\
\hline
\end{tabular}

Data are presented as mean \pm SD unless otherwise stated SD, standard deviation 
(total of 73/84; Italy, 44/49; Belgium, 7/10; UK, $22 / 25$ ) and, in most patients, the previous FIX product was the standard-acting rFIX nonacog alfa. Of note, 11 paediatric patients were included in the analysis, 10 from Italy and 1 from the UK.

The mean duration of observation $[ \pm$ standard deviation (SD)] of rIX-FP treatment in Italy, Belgium and the UK was $46.3 \pm 34.7$, $19.2 \pm 11.3$ and $29.7 \pm 18.1$ weeks, respectively, and the duration of treatment analysed on prior FIX treatment was 52 weeks for all patients.

\section{Haemostatic Efficacy}

Data for bleeding events from patients switching from prior FIX prophylaxis to rIX-FP prophylaxis were available for $73 / 84$ (86.9\%) patients from Italy, Belgium and the UK (Table 2). Of the 11 paediatric patients enrolled in the study, the patients from Italy $(n=10)$ all switched from prior FIX prophylaxis to rIX-FP prophylaxis.

Overall, the switch to prophylaxis with rIXFP led to reductions in mean ABR of $94.3 \%$ in Italy, $93.9 \%$ in Belgium and $67.7 \%$ in the UK compared with prophylaxis with previous FIX product (Table 2 ). In addition, the proportions of patients with zero bleeds or zero spontaneous bleeds were much higher with rIX-FP prophylaxis than with prior FIX prophylaxis $(6 \times$,
$5 \times$ and $\geq 36 \times$ higher for zero bleeds; $2 \times$, $2 \times$ and $3 \times$ higher for zero spontaneous bleeds) in Italy, Belgium and the UK, respectively (Table 2).

For the Italian cohort, paediatric patients experienced a reduction in mean (SD) ABR/ AsBR following the switch from prior FIX to rIXFP $[5.6(11.6) / 0.1(0.3)$ to $0.3(0.5) / 0.0(0.0)$, respectively] and an increase in the proportion of patients with zero bleeds or zero spontaneous bleeds $(1.75 \times$ and $1.1 \times$ higher, respectively). In patients $\geq 12$ years in Italy $(n=34)$, mean ABR/AsBR (SD) was reduced from 2.8 (2.0)/0.9 (1.1) to $0.1(0.3) / 0.03(0.2)$ following the switch to rIX-FP; these patients also experienced an increase in the proportion of patients with zero bleeds or zero spontaneous bleeds following the switch to rIX-FP $(14.9 \times$ and $2.5 \times$ higher, respectively).

\section{Dosing Frequency}

Dosing regimens in patients receiving prior FIX prophylaxis who switched to rIX-FP prophylaxis in Italy, Belgium and the UK are shown in Fig. 1 . Only $7 / 73$ patients $(9.6 \%)$ received prophylaxis with their prior FIX product once weekly but, after switching to rIX-FP prophylaxis, the majority of patients had a reduction in dosing frequency: $61 / 73$ patients $(83.6 \%)$ receiving rIX-FP every $7-12$ days, $9 / 73$ patients $(12.3 \%)$ receiving rIX-FP once every 2 weeks and

Table 2 Dosing, consumption and bleeding rates with rIX-FP prophylaxis and prior FIX product prophylaxis in patients with haemophilia B in Italy, Belgium and the UK

\begin{tabular}{|c|c|c|c|c|c|c|}
\hline & \multicolumn{2}{|l|}{ Italy } & \multicolumn{2}{|l|}{ Belgium } & \multicolumn{2}{|l|}{ UK } \\
\hline & $\begin{array}{l}\text { rIX-FP } \\
(n=44)\end{array}$ & $\begin{array}{l}\text { Prior FIX } \\
(n=44)\end{array}$ & $\begin{array}{l}\text { rIX-FP } \\
(n=7)\end{array}$ & $\begin{array}{l}\text { Prior FIX } \\
(n=7)\end{array}$ & $\begin{array}{l}\text { rIX-FP } \\
(n=22)\end{array}$ & $\begin{array}{l}\text { Prior FIX } \\
(n=22)\end{array}$ \\
\hline Dose, IU/kg/week & $38.6 \pm 16.5$ & $84.4 \pm 34.4$ & $30.3 \pm 11.5$ & $103.1 \pm 60.9$ & $29.2 \pm 8.5$ & $71.7 \pm 18.9$ \\
\hline$A B R$ & $0.2 \pm 0.4$ & $3.5 \pm 5.7$ & $0.4 \pm 0.8$ & $6.6 \pm 3.4$ & $1.0 \pm 0.9$ & $3.1 \pm 1.4$ \\
\hline AsBR & $0.02 \pm 0.2$ & $0.7 \pm 1.0$ & $0.0 \pm 0.0$ & $3.0 \pm 3.3$ & $0.4 \pm 0.6$ & $2.0 \pm 1.5$ \\
\hline Patients with zero bleeds, \% & 84.1 & 13.6 & 71.4 & 14.3 & 36.4 & 0.0 \\
\hline $\begin{array}{l}\text { Patients with zero } \\
\text { spontaneous bleeds, \% }\end{array}$ & 97.7 & 50.0 & 100.0 & 42.9 & 63.6 & 22.7 \\
\hline
\end{tabular}

Data are presented as mean \pm SD unless otherwise stated

$\mathrm{ABR}$, annualised bleeding rate; AsBR, annualised spontaneous bleeding rate; $\mathrm{SD}$, standard deviation 

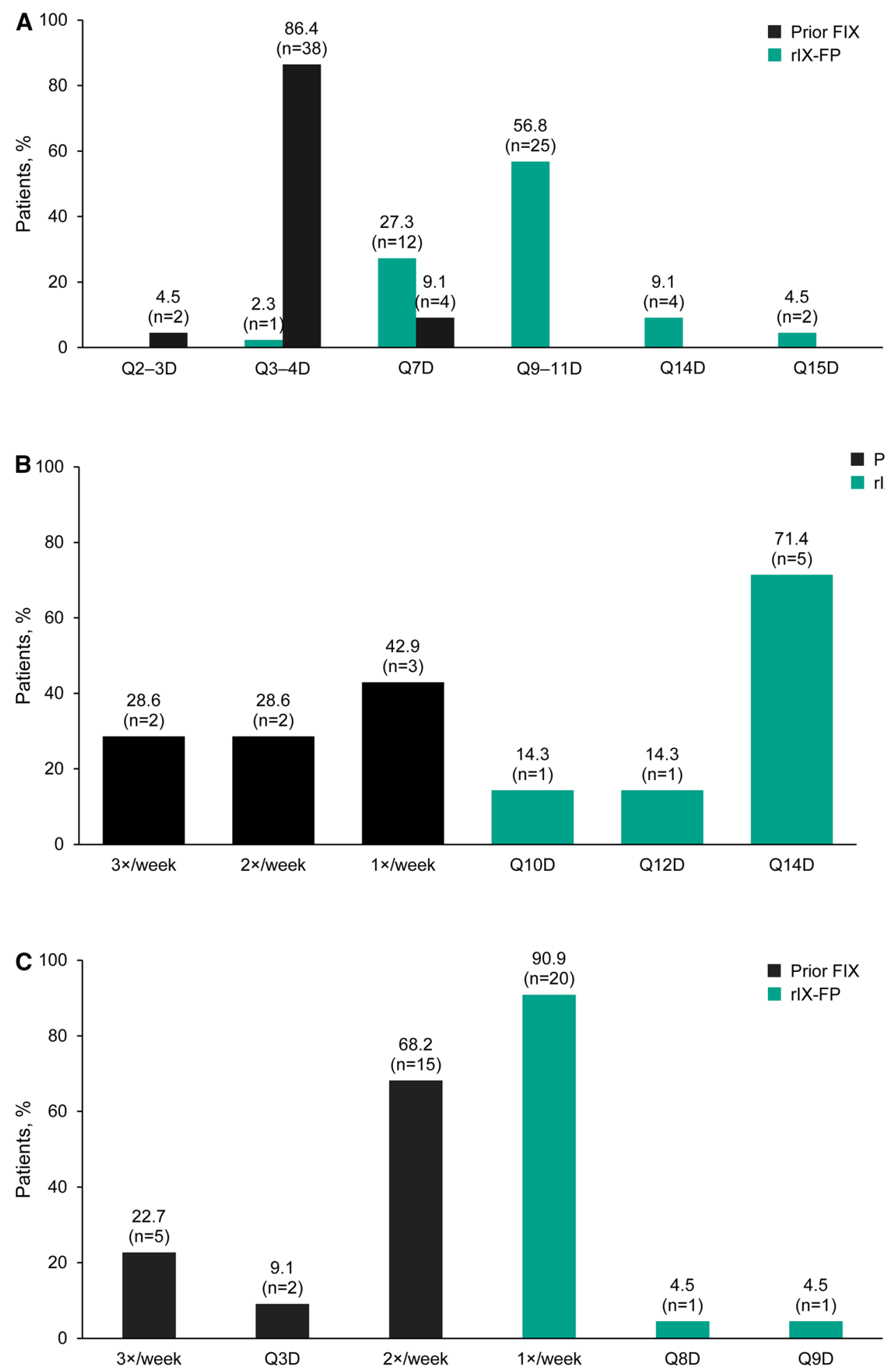

Fig. 1 Dosing regimens in patients receiving prior FIX prophylaxis who switched to rIX-FP prophylaxis in Italy (a), Belgium (b) and the UK (c). Results should be interpreted with caution because of low patient numbers 
2/73 patients (2.7\%) every 15 days (Fig. 1 ). Similar results were observed in paediatric patients, with 8/10 (80\%) patients previously infusing every 3-4 days and after switching to rIX-FP 9/10 (90\%) were dosing every 7 days or less including three patients who were infusing every 14-15 days with rIX-FP. In adult patients, $5.9 \%$ received 7 -day prophylaxis with their prior product; following the switch to rIX-FP, all patients dosed once every 7 days or less.

\section{Consumption}

Mean weekly FIX doses before and after switching to prophylaxis with rIX-FP in patients from Italy, Belgium and the UK are shown in Table 2. The switch to prophylaxis with rIX-FP was associated with reductions in mean weekly FIX consumption of 54\%, 71\% and 59\% in Italy, Belgium and the UK, respectively, compared with prior FIX prophylaxis. In paediatric patients, mean weekly consumption was consistently lower for patients treated with rIX-FP prophylaxis (44.1 IU/kg/week) compared with prior FIX prophylaxis (106.5 IU/kg/week), a $58 \%$ reduction in factor consumption. Results were similar when considering adult patients alone who were previously treated with nonacog alfa prophylaxis (86.8 IU/ kg/week) who then switched to rIX-FP prophylaxis (39.7 IU/ $\mathrm{kg} /$ week), a $54 \%$ reduction in factor consumption.

A simple cost per success analysis using PriceCentric as a data source revealed that rIXFP showed the lowest cost per success vs. nonacog alfa in Italy (-29\%), Belgium (-55\%) and the UK (-50\%); success was defined as patients experiencing no spontaneous bleeds. This continued to be true when the price of nonacog alfa was reduced by $20 \%$ or when the efficacy of rIXFP was reduced by $20 \%$. Similar results were also shown if success was instead defined as patients with zero ABR instead of zero AsBR.

\section{DISCUSSION}

This is the first analysis to assess the real-world clinical benefits of switching to prophylaxis with rIX-FP from a prior SHL product in Italy,
Belgium and the UK. The mean ABR was $>3$ in all of the countries prior to switching and was $\leq 1$ in all three countries following the switch to prophylaxis with rIX-FP, amounting to mean reductions of $\geq 2$ bleeding episodes per year. Moreover, the proportions of patients with zero bleeds increased markedly, and the proportions of patients with zero spontaneous bleeds increased at least two-fold, following the switch to prophylaxis with rIX-FP. The observation that there were no UK patients reporting zero bleeds prior to the switch to rIX-FP prophylaxis may have been a reflection of the high proportion of UK patients with severe haemophilia B, although there was also a high proportion of patients with severe disease in Italy. In addition, ABRs reported in the paediatric patients switching from prophylaxis with their prior product to prophylaxis with rIX-FP were 5.6 and 0.3 , respectively; thus, both adult and paediatric patients demonstrated a similar reduction in $A B R$ and AsBR values after switching.

Patients had a reduced frequency of infusions following the switch to prophylaxis with rIX-FP compared with prophylaxis with prior FIX. However, changes in the infusion frequency following the switch from prior prophylaxis to rIX-FP varied between countries, with $13.6 \%$ of patients in Italy and $71.4 \%$ of patients in Belgium receiving rIX-FP infusions at intervals of $\geq 14$ days after switching. In the UK, $90.9 \%$ of patients who switched to prophylaxis with rIX-FP received once-weekly prophylaxis, which was in line with published guidance on prophylaxis with EHL coagulation factor concentrates from the UK Haemophilia Centres Doctors' Organisation [19]. Reduced dosing frequency may improve treatment adherence and has the potential to improve treatment experience by reducing treatment burden [20]. The higher trough levels achieved with rIX-FP also have the potential to improve patient management and allow the patient to live a more active lifestyle [21]. While treatment efficacy should always remain the most important outcome when selecting a treatment regimen, the enhanced features of rIX-FP are allowing patients with haemophilia the opportunity to live a healthier and less burdensome life [20]. 
This analysis determined that factor consumption was reduced on average by $2.8 \times$ (range 2.2-3.7 $\times$ ) in all patients with haemophilia B who switched to prophylaxis with rIXFP compared with prophylaxis with their prior FIX product in Italy, Belgium and the UK. The reductions in factor consumption were particularly marked in Belgium and were accompanied by the longest dosing intervals among the three countries under consideration; however, the sample size in this country was limited $(n=7)$. Similar reductions were seen when considering paediatric patients alone, consumption reduced by $2.4 \times$ after switching to rIX-FP, although the results should be interpreted with caution because of the small sample size.

A complex health economic evaluation was outside the scope of this work; however, a simple cost per success analysis allowed consideration of the clinical benefits of rIX-FP as well as cost per unit. It is important to note that there are some challenges when calculating the true annual factor consumption cost with prior FIX and rIX-FP in this analysis, as patients switched from various FIX products (plasma and recombinant) and list prices vary within countries and centres, hence making general assumptions in this cohort of patients is difficult. However, the estimated reduced cost per success of rIX-FP alongside the reduced consumption and increased proportion of patients with zero bleeds discussed in this analysis demonstrates the numerous potential benefits of rIX-FP in patients with haemophilia $B$.

The EHL FIX products received their marketing authorisations in the EU in May 2016 (rIX-FP and rFIXFc) and June 2017 (N9-GP); therefore, this study only evaluated switching from SHL products to rIX-FP. As further data become available over time, it would be interesting to compare clinical outcomes in patients with haemophilia B switching between the different EHL FIX products.

Evidence of the clinical benefits of switching from SHL to EHL FIX replacement products in patients with haemophilia B derived from realworld studies is scarce. A previous study from the USA focused on cost analysis rather than on the potential clinical benefits to patients [22].
However, our findings of reduced bleeding and factor consumption following the switch to rIXFP prophylaxis were consistent with a retrospective review of existing medical records of 19 patients with haemophilia B in the USA who had been prescribed rFIXFc [23]. This analysis found that ABR and AjBR fell from 2.5 and 2.1 to 0.82 and 0.37 , respectively, following the initiation of the EHL factor treatment. Overall, it was concluded that treatment with EHL factors reduced factor consumption by nearly $50 \%$ compared with treatment with SHL factors in patients with haemophilia B [23]. In addition, preliminary data from a European survey of 33 haemophilia treatment centres that had switched to using EHL FVIII and FIX products (rFVIIIFc, rFIXFc and rIX-FP) have indicated that the number of infusions was reduced by $>30 \%$ after switching SHL to EHL replacement products, with bleeding episodes reduced by $\leq 50 \%$ at $86 \%(18 / 21)$ of responding centres [24].

Retrospective chart review is widely used in healthcare research, but the sampling strategies used may present limitations in terms of the generalisability of results [25]. This analysis was subject to a number of limitations; for example, the assessment period was short and, in order to not overburden the contributing centres, only information needed to meet the objectives of the study were requested; as such, detailed clinical information regarding the patient population and location, severity, treatment and outcomes of bleeding events was lacking. In addition, only patients who switched to rIX-FP from a prior replacement product were considered and this may not be representative of the entire patient population. Information on reasons for switching products was also absent and there was potential for selection bias as not all centres contributed all of their patient data; thus, these data may not be representative of the entire patient population.

Irrespective of these limitations, the results of this analysis of switching from prophylaxis with a SHL FIX product to rIX-FP were consistent with the experience in rIX-FP clinical trials $[13,14]$, in that prophylaxis with rIX-FP may lead to improved bleeding control, reduced dose frequency and lower prophylactic factor 
consumption in real-world use. Further research in prospective studies with larger sample sizes, including adult and paediatric patients, with more understanding of patient characteristics (e.g., joint status, PK parameters, etc.) is needed to strengthen the data identified here and to detect treatment patterns in patients with haemophilia B.

\section{CONCLUSIONS}

In conclusion, this retrospective review of realworld evidence demonstrated that switching to rIX-FP from prior FIX was associated with improved haemostatic efficacy and reduced factor consumption in patients with haemophilia B from Italy, Belgium and the UK. This real-world analysis further strengthens the rIXFP clinical trial data, showing that the effective bleeding prevention demonstrated in clinical trials is consistent with that seen in real-world clinical practice.

\section{ACKNOWLEDGEMENTS}

Funding. Sponsorship for this study, the Rapid Service Fee and Open Access Fee were funded by CSL Behring. All authors had full access to all of the data in this study and take complete responsibility for the integrity of the data and accuracy of the data analysis.

Medical Writing Assistance. Editorial assistance in the preparation of this article was provided by Meridian HealthComms Ltd., Plumley, Cheshire, UK, funded by CSL Behring.

Authorship. All named authors meet the International Committee of Medical Journal Editors (ICMJE) criteria for authorship for this article, take responsibility for the integrity of the work as a whole and have given their approval for this version to be published.

Prior Presentation. Marino et al. Presented at the XXVII Congress of the International Society on Thrombosis and Haemostasis, July
6-10, 2019; Marino et al. Presented at the 13th Annual Congress of the European Association for Haemophilia and Allied Disorders, 5-7 February 2020.

Disclosures. Cedric Hermans: Member of advisory committee and speaker bureau (Bayer, Shire, Pfizer, Novo Nordisk, Octapharma, SOBI BIOGEN, Roche, CSL Behring, LFB, Biomarin, CAF-DCF), research grants (Bayer, Shire, Pfizer, NovoNordisk, Octapharma, SOBI, CSL Behring). Catherine Lambert: Consultancy for CSL Behring, Bayer, Pfizer, Roche, Novo Nordisk, Sobi and Sanofi. Sarah Mangles: Consultancy for Roche, Takeda, Novo Nordisk, funding to attend meetings from SOBI, Octapharma, Novo Nordisk, Shire and Roche/Chugai. Patrick Sommerer and Vincent Rives are employees of CSL Behring. Geraldine Maro is an employee of Adivo Associates. Giuseppe Malcangi and Renato Marino have nothing to disclose.

Compliance with Ethics Guidelines. The study obtained only pre-existing data from patients who were never identified to researchers, and therefore informed consent was not required. The study was submitted to an Institutional Review Board and was determined to be exempt under category 4 'Secondary research for which consent is not required'.

Data Availability. CSL will only consider requests to share Individual Patient Data (IPD) that are received from systematic review groups or bona-fide researchers. CSL will not process or act on IPD requests until 12 months after article publication on a public website. An IPD request will not be considered by CSL unless the proposed research question seeks to answer a significant and unknown medical science or patient care question. Applicable countryspecific privacy and other laws and regulations will be considered and may prevent sharing of IPD. Requests for use of the IPD will be reviewed by an internal CSL review committee. If the request is approved, and the researcher agrees to the applicable terms and conditions in a data sharing agreement, IPD that has been appropriately anonymised will be made available. Supporting documents including study 
protocol and Statistical Analysis Plan will also be provided. For information on the process and requirements for submitting a voluntary data sharing request for IPD, please contact CSL at clinicaltrials@cslbehring.com.

Open Access. This article is licensed under a Creative Commons Attribution-NonCommercial 4.0 International License, which permits any non-commercial use, sharing, adaptation, distribution and reproduction in any medium or format, as long as you give appropriate credit to the original author(s) and the source, provide a link to the Creative Commons licence, and indicate if changes were made. The images or other third party material in this article are included in the article's Creative Commons licence, unless indicated otherwise in a credit line to the material. If material is not included in the article's Creative Commons licence and your intended use is not permitted by statutory regulation or exceeds the permitted use, you will need to obtain permission directly from the copyright holder. To view a copy of this licence, visit http://creativecommons.org/licenses/by$\mathrm{nc} / 4.0 /$.

\section{REFERENCES}

1. Srivastava A, Brewer AK, Mauser-Bunschoten EP, et al. Guidelines for the management of hemophilia. Haemophilia. 2013;19(1):e1-47.

2. Manco-Johnson MJ, Abshire TC, Shapiro AD, et al. Prophylaxis versus episodic treatment to prevent joint disease in boys with severe hemophilia. N Engl J Med. 2007;357(6):535-44.

3. Oldenburg J. Optimal treatment strategies for hemophilia: achievements and limitations of current prophylactic regimens. Blood. 2015;125(13): 2038-44.

4. Hacker MR, Geraghty S, Manco-Johnson M. Barriers to compliance with prophylaxis therapy in haemophilia. Haemophilia. 2001;7(4):392-6.

5. Powell JS, Pasi KJ, Ragni MV, et al. Phase 3 study of recombinant factor IX Fc fusion protein in hemophilia B. N Engl J Med. 2013;369(24):2313-23.
6. Saxena K. Barriers and perceived limitations to early treatment of hemophilia. J Blood Med. 2013;4: 49-56.

7. De Moerloose P, Urbancik W, Van Den Berg HM, Richards M. A survey of adherence to haemophilia therapy in six European countries: results and recommendations. Haemophilia. 2008;14(5):931-8.

8. Thornburg CD, Duncan NA. Treatment adherence in hemophilia. Patient Prefer Adherence. 2017;11: 1677-86.

9. Castaman G. The benefits of prophylaxis in patients with hemophilia B. Expert Rev Hematol. 2018;11(8):673-83.

10. von Mackensen S, Kalnins W, Krucker J, et al. Haemophilia patients' unmet needs and their expectations of the new extended half-life factor concentrates. Haemophilia. 2017;23(4):566-74.

11. World Federation of Hemophilia. Current issues in prophylaxis. 2014. https://www.wfh.org/en/abd/ prophylaxis/current-issues-in-prophylaxis. Accessed 8 July 2019.

12. Lambert T, Benson G, Dolan G, et al. Practical aspects of extended half-life products for the treatment of haemophilia. Ther Adv Hematol. 2018;9(9):295-308.

13. Kenet $\mathrm{G}$, Chambost $\mathrm{H}$, Male $\mathrm{C}$, et al. Long-acting recombinant fusion protein linking coagulation factor IX with albumin (rIX-FP) in children. Results of a phase 3 trial. Thromb Haemost. 2016;116(4): 659-68.

14. Santagostino E, Martinowitz U, Lissitchkov T, et al. Long-acting recombinant coagulation factor IX albumin fusion protein (rIX-FP) in hemophilia B: results of a phase 3 trial. Blood. 2016;127(14): 1761-9.

15. Fischer K, Kulkarni R, Nolan B, et al. Recombinant factor IX Fc fusion protein in children with haemophilia B (Kids B-LONG): results from a multicentre, non-randomised phase 3 study. Lancet Haematol. 2017;4(2):e75-82.

16. Powell JS, Apte S, Chambost $\mathrm{H}$, et al. Long-acting recombinant factor IX Fc fusion protein (rFIXFc) for perioperative management of subjects with haemophilia B in the phase 3 B-LONG study. Br J Haematol. 2015;168(1):124-34.

17. Carcao M, Zak M, Abdul Karim F, et al. Nonacog beta pegol in previously treated children with hemophilia B: results from an international openlabel phase 3 trial. J Thromb Haemost. 2016;14(8): 1521-9. 
18. Collins PW, Young G, Knobe K, et al. Recombinant long-acting glycoPEGylated factor IX in hemophilia B: a multinational randomized phase 3 trial. Blood. 2014;124(26):3880-6.

19. Collins P, Chalmers E, Chowdary P, et al. The use of enhanced half-life coagulation factor concentrates in routine clinical practice: guidance from UKHCDO. Haemophilia. 2016;22(4):487-98.

20. von Mackensen S, Shah J, Seifert W, Kenet G. Health-related quality of life in paediatric haemophilia B patients treated with rIX-FP. Haemophilia. 2019;25(1):45-53.

21. Gill JC, Roberts J, Li Y, Castaman G. Sustained high trough factor IX activity levels with continued use of rIX-FP in adult and paediatric patients with haemophilia B. Haemophilia. 2019;25(3): e219-e222.

22. Tortella BJ, Alvir J, McDonald M, et al. Real-world analysis of dispensed IUs of coagulation factor IX and resultant expenditures in hemophilia B patients receiving standard half-life versus extended half-life products and those switching from standard half-life to extended half-life products. J Manag Care Spec Pharm. 2018;24(7):643-53.

23. Wang C, Young G. Clinical use of recombinant factor VIII Fc and recombinant factor IX Fc in patients with haemophilia A and B. Haemophilia. 2018;24(3):414-9.

24. Peyvandi F, Garagiola I, Boscarino M, Ryan A, Hermans C, Makris M. Real-life experience in switching to new extended half-life products at European haemophilia centres. Haemophilia. 2019;25(6):946-52.

25. Vassar M, Holzmann M. The retrospective chart review: important methodological considerations. J Educ Eval Health Prof. 2013;10:12. 